Swati Tyagi (Rupnagar)

Syed AbBas (Mandi)

Manuel Pinto (Santiago)

\title{
GLOBAL EXPONENTIAL STABILITY IN LAGRANGE SENSE FOR PERIODIC NEURAL NETWORKS WITH VARIOUS ACTIVATION FUNCTIONS AND TIME-VARYING DELAYS
}

Abstract. In recent years, the concept of Lyapunov stability has received a remarkable attention in the field of neural networks. However the stability in Lagrange sense for neural networks has not been studied much. It is to be noticed that while Lyapunov stability refers to stability of the equilibrium point, Lagrange stability refers to the stability of the total system. In this paper, we study the global exponential stability in Lagrange sense for periodic neural networks with multiple time delays and more general activation functions including general bounded and sigmoidal type activation functions. By constructing suitable Lyapunov-like functions, we provide easily verifiable criteria for the boundedness and global exponential attractivity of periodic neural networks. We present a detailed estimation of global exponential attractive sets from the system parameters without any supposition on existence. We investigate whether the equilibrium point of the network system is globally exponentially stable by means of globally exponentially attractive sets. At the end, we give some numerical examples to validate our analytical findings. The results obtained are helpful in designing globally asymptotically stable cellular neural networks and reduce the search domain of optimization.

2010 Mathematics Subject Classification: 92B20, 34D23, 34D99, 35B35.

Key words and phrases: periodic neural network, Lagrange stability, global exponential attractivity, time delays, Lyapunov-like functions.

Received 25 September 2016; revised 15 September 2017, 8 May 2018 and 17 July 2018. Published online 12 April 2019. 
1. Introduction. Recurrent neural networks (RNN's) have found numerous applications since the pioneering work of Hopfield [8]. From the system-theoretic point of view, because of the special non-linear structure of neural networks, their global stability becomes of interest. When using RNN's to solve optimization, control or signal processing problems, Lyapunov global stability is one of the most desirable properties. From the dynamical point of view, globally stable networks in Lyapunov sense are monostable systems, which restrict each neural network to one equilibrium point. Many authors have investigated the dynamic behaviour of various systems using the concept of Lyapunov stability. For instance, we refer to [1, 2, 17, 5, 3, 6, 19, 18] and references cited therein. Though monostable neural networks have been found to be computationally restrictive, multistable dynamics are essential in many applications. In such cases, neural networks are no longer globally stable and more appropriate notions of stability are needed.

One can view the global stability in Lyapunov sense as a special case of stability in Lagrange sense by regarding the equilibrium point as an attracting set [13. Recently, many researchers have studied global exponential stability in Lagrange sense for various neural network models with different time delays. For more details, we refer to [16, 15, 21, 20, 14, 23] and references cited therein. In [26], the authors considered boundedness, attractivity and complete convergence as three basic properties of a multistable network. Moreover global exponential stability in Lagrange sense is of much significance, as discussed below.

The field of neural networks is widely used in optimization computation to find the equilibrium point of a neural network. Through the concept of stability in the Lagrange sense, we can easily find a globally exponentially attractive set and thus we obtain a rough boundary of the optimization solution set. Moreover, in dynamic systems, for the existence of periodic and almost periodic solutions, the system should be ultimately bounded. The periodic and almost periodic solutions must be within the global attractive set, which is achieved through stability in Lagrange sense [7. Also it is generally considered that a continuous dynamical system exhibits chaos if the system is dissipative (or ultimately bounded) on large scale with positive Lyapunov index on small scale [4]. In recent years, researchers have found chaos in neural networks also. Thus it becomes important to study stability in Lagrange sense. Furthermore, if we consider our equilibrium point as an attractive set, then the global stability in Lyapunov sense at the unique equilibrium point can be taken as a special case of stability in the Lagrange sense. Hence from both practical and theoretical perspective, it becomes significant to study the global stability in Lagrange sense for neural networks. To date, a series of results for periodic neural networks have been obtained 
(for instance, see [10, 12, 25, 24, 11, 9, 27, 22] and references cited therein). While analyzing stability of a neural network model, characteristics of activation functions and network parameters play an important role, since the conditions to be imposed on the neural network are determined from them. Consequently, studying neural networks with general activation functions becomes important.

Motivated by the above discussion, in this paper, our objective is to study the global exponential stability in Lagrange sense for a general periodic neural network. In [22, the authors have studied global exponential stability in Lagrange sense for periodic neural networks by considering bounded and Lurie-type activation functions with discrete time delay. Our results complement the results obtained in [22] and improve them in the sense that we now take into account time-varying time delays along with activation functions of bounded and sigmoidal type.

The organization of this paper is as follows. In Section 2, we give the notion of globally exponentially attractive (GEA) sets and global exponential stability (GES) in Lagrange sense along with one preliminary result; these are used in later sections to prove the main results. In Sections 3 and 4. we derive stability of a neural network model in Lagrange sense for various types of activation functions. In Section 5 , we give some numerical examples to validate our results. Finally, in Section 6 we give some concluding results.

2. Preliminaries. Consider the periodic neural network (PNN) model with different activation functions and multiple time-varying delays described by the equation

$$
\begin{aligned}
\frac{d x_{i}(t)}{d t}= & -d_{i}(t) x_{i}(t)+\sum_{j=1}^{n} a_{i j}(t) f_{j}\left(x_{j}(t)\right) \\
& +\sum_{j=1}^{n} b_{i j}(t) g_{j}\left(x_{j}\left(t-\tau_{i j}(t)\right)\right)+I_{i}(t),
\end{aligned}
$$

where $i=1, \ldots, n, x_{i}(t)$ is the state variable of the $i$ th neuron, $a_{i j}(t)$ and $b_{i j}(t)$ are connection weights from neuron $i$ to neuron $j, I_{i}(t) \in C(\mathbb{R}, \mathbb{R})$ is the external bias, $\tau_{i j}(t)$ represents the transmission delay and $\tau_{i j}(t) \in[0, \tau]$, $i, j=1, \ldots, n$, where $\tau>0$ is constant, $f_{j} \in C(\mathbb{R}, \mathbb{R})$ and $g_{j} \in C(\mathbb{R}, \mathbb{R})$ are non-linear activation functions. The initial condition associated with network 2.1) takes the form

$$
x_{i}(s)=\phi_{i}(s), \quad s \in[-\tau, 0], i=1, \ldots, n,
$$

where $\phi(s)=\left(\phi_{1}(s), \ldots, \phi_{n}(s)\right)^{T}$ is a continuous function defined on $[-\tau, 0]$. 
When $b_{i j}(t)=0$, the PNN (2.1) reduces to

$$
\frac{d x_{i}(t)}{d t}=-d_{i}(t) x_{i}(t)+\sum_{j=1}^{n} a_{i j}(t) f_{j}\left(x_{j}(t)\right)+I_{i}(t) .
$$

For $a_{i j}(t)=0,2.1$ takes the form

$$
\frac{d x_{i}(t)}{d t}=-d_{i}(t) x_{i}(t)+\sum_{j=1}^{n} b_{i j}(t) g_{j}\left(x_{j}\left(t-\tau_{i j}(t)\right)\right)+I_{i}(t) .
$$

The cases 2.3) and (2.4) are particular cases of 2.1), which are discussed further. Throughout, we assume $d_{i}(t), a_{i j}(t), b_{i j}(t), I_{i}(t), \tau_{i j}(t)$ are continuous $\omega$-periodic functions, that is, $d_{i}(t+\omega)=d_{i}(t)$ for all $t$ etc.

2.1. Notations and assumptions. Let $C$ denote the Banach space of continuous functions $\psi:[-\tau, 0] \rightarrow \mathbb{R}^{n}$ equipped with the norm $\|\psi\|=$ $\sup _{s \in[-\tau, 0]}|\psi(s)|$. Let $\mathcal{C}_{\mathcal{F}}^{+}$be the set of all continuous functionals $K: C \rightarrow$ $[0, \infty)$ mapping bounded sets in $C$ into bounded sets in $[0, \infty)$. For a given constant $\mathcal{T}>0$, the subset $C_{\mathcal{T}}$ is defined as $\{\psi \in C|| \psi \mid \leq \mathcal{T}\}$. For any initial condition $\sigma \in C$, solutions of (2.1) starting from $\sigma$ will be denoted as $x(t ; \sigma)$. If there is no need to emphasize the initial condition, any solution of (2.1) will be denoted by $x(t)$. For any continuous bounded function $h(t)$, $t \geq 0$, we write $|\bar{h}|=\sup _{t \geq 0}|h(t)|$ and $|\underline{h}|=\inf _{t \geq 0}|h(t)|$.

We define three classes of activation functions for the neural network model (2.1). Consider the set of non-decreasing functions

$$
\mathcal{K}=\left\{\phi \in C(\mathbb{R}, \mathbb{R}) \mid s \phi(s)>0, \forall s \neq 0, D^{+} \phi(s) \geq 0, \forall s \in \mathbb{R}\right\},
$$

where $D^{+} \phi(s)$, the right upper Dini derivative of $\phi$, is given by

$$
D^{+} \phi(s)=\limsup _{h \rightarrow 0^{+}} \frac{\phi(s+h)-\phi(s)}{h} .
$$

First, we consider the class of sigmoidal functions, defined by

$$
\mathcal{S}=\{g \in C(\mathbb{R}, \mathbb{R})|g(0)=0, \exists k>0,| g(x) \mid \leq k, \forall x \in \mathbb{R}\},
$$

where the constant $k$ is called a saturation constant.

The second class of activation functions consists of continuous nondecreasing activation functions vanishing at zero:

$$
\mathcal{G}=\{g \in \mathcal{K} \mid g(0)=0\} .
$$

The third class of activation functions consists of bounded functions:

$$
\mathcal{B}=\{g \in \mathcal{K}|\exists k>0,| g(x) \mid \leq k, \forall x \in \mathbb{R}\} .
$$

2.2. Definitions and lemmas. Let $\Omega \subset \mathbb{R}^{n}$ be a compact set. We denote its complement by $\mathbb{R}^{n} \backslash \Omega$, and $\rho(x, \Omega)=\inf _{y \in \Omega}|x-y|$ denotes the distance from $x \in \mathbb{R}^{n}$ to $\Omega$. 
Definition 2.1 (22]). The neural network model (2.1) is said to be uniformly stable in Lagrange sense (or uniformly bounded) if for any $\mathcal{L}>0$, there exists a constant $K=K(\mathcal{L})$ such that $|x(t ; \phi)|<K$ for all $\phi \in \mathcal{C}_{\mathcal{L}}$ and $t \geq 0$.

Definition $2.2([22])$. A compact set $\Omega \subset \mathbb{R}^{n}$ is said to be a globally attractive set of the neural network (2.1) if for every solution $x(t) \in \mathbb{R}^{n}$, $t \geq 0$, we have

$$
\lim _{t \rightarrow+\infty} \rho(x(t), \Omega)=0 .
$$

Definition $2.3([22])$. Assume there exists a radially unbounded and positive definite function $V(x)$, a continuous functional $K \in \mathcal{C}_{\mathcal{F}}^{+}$, and positive constants $l$ and $\alpha$ such that for any solution $x(t)=x(t ; \psi)$ of (2.1),

$$
V(x(t))>l, t \geq 0, \quad \text { implies } V(x(t))-l \leq K(\psi) e^{-\alpha t}, \forall t \geq 0 .
$$

Then the neural network (2.1) is said to be globally exponentially attractive with respect to $V$, and the compact set $\Omega=\left\{x \in \mathbb{R}^{n} \mid V(x) \leq l\right\}$ is called a globally exponentially attractive (GEA) set of (2.1).

Definition 2.4 ([22]). Suppose there exist radially unbounded and positive definite functions $V_{i}(x), x \in \mathbb{R}$, continuous functionals $K_{i} \in \mathcal{C}_{\mathcal{F}}^{+}$, and positive constants $l_{i}$ and $\alpha_{i}$ such that for any solution $x(t)=x(t ; \psi)$ of 2.1),

$$
V_{i}\left(x_{i}(t)\right)>l_{i}, \forall t \geq 0, \quad \text { implies } \quad V_{i}\left(x_{i}(t)\right)-l_{i} \leq K_{i}(\psi) e^{-\alpha_{i} t}, \forall t \geq 0 .
$$

Then the neural network (2.1) is called globally exponentially attractive with respect to $\left(V_{1}, \ldots, V_{n}\right)$, and the compact set $\Omega=\left\{x \in \mathbb{R}^{n} \mid V_{i}\left(x_{i}\right) \leq l_{i}, \forall i\right\}$ is called a globally exponentially attractive set of (2.1).

Definition 2.5 ([22]). The neural network (2.1) is said to be globally exponentially stable (GES) in Lagrange sense if it is both uniformly stable in Lagrange sense and globally exponentially attractive. If we need to emphasize the Lyapunov-like functions, we say that the network is globally exponentially stable in Lagrange sense with respect to $V$ or $\left(V_{1}, \ldots, V_{n}\right)$.

Obviously, if the network (2.1) has a global attractive set, it is uniformly bounded; and if the network (2.1) has a GEA set, it is GES in Lagrange sense. Next we give a lemma, which is used in the proofs of our main results.

Lemma $2.6([22])$. Let $G \in C\left(\left[t_{0}, \infty\right), \mathbb{R}\right)$, and suppose there exist constants $\alpha \neq 0$ and $\beta$ such that

$$
D^{+} G(t) \leq-\alpha G(t)+\beta, \quad t \geq t_{0}
$$

Then

$$
G(t)-\frac{\beta}{\alpha} \leq\left(G\left(t_{0}\right)-\frac{\beta}{\alpha}\right) \exp \left\{-\alpha\left(t-t_{0}\right)\right\}, \quad t \geq t_{0} .
$$


In particular, if $G(t) \geq \beta / \alpha$ for all $t \geq t_{0}$, then $G(t)$ exponentially approaches $\beta / \alpha$ as $t$ increases.

3. Global exponential stability for $f_{i} \neq g_{i}$. In this section, we derive stability results for different activation functions, that is, $f_{i} \neq g_{i}$. We first consider the case of bounded activation functions and later analyze the results for $f_{i}$ of sigmoidal type and $g_{i}$ bounded.

3.1. Bounded activation functions. In this subsection, we consider the network (2.1) with bounded feedback functions $f_{i}, g_{i}(i=1, \ldots, n)$. We further assume that the network satisfies all the assumptions of the previous section. Let $k_{i}, h_{i}>0$ be the saturation constants of $g_{i}$ and $f_{i}$. For $i=1, \ldots, n$, define

$$
\begin{aligned}
& M_{i}^{(1)}=\frac{1}{2}\left(\sum_{j=1}^{n}\left|\bar{a}_{i j}\right| h_{j}+\sum_{j=1}^{n}\left|\bar{b}_{i j}\right| k_{j}+\left|\bar{I}_{i}\right|\right), \\
& M_{i}^{(2)}=\frac{1}{2}\left(\sum_{j=1}^{n}\left|\bar{a}_{i j}\right| h_{j}+\left|\bar{I}_{i}\right|\right) \\
& M_{i}^{(3)}=\frac{1}{2}\left(\sum_{j=1}^{n}\left|\bar{b}_{i j}\right| k_{j}+\left|\bar{I}_{i}\right|\right) .
\end{aligned}
$$

Now we state our result.

THEOREM 3.1. Assume that the activation functions $f_{i}, g_{i}$ are all bounded, $f_{i}, g_{i} \in \mathcal{B},\left|f_{i}\right| \leq h_{i}$ and $\left|g_{i}\right| \leq k_{i}$, where $h_{i}, k_{i}>0$ are constants and $x_{i} f_{i}\left(x_{i}\right)>0$ for $x_{i} \neq 0, f_{i}(0)=0, i=1, \ldots, n$, and let $\left|d_{i}\right|=\inf _{t \geq 0}\left|d_{i}(t)\right|$. Then the PNN (2.1) is globally exponentially stable in Lagrange sense with globally exponentially attractive sets

$$
\begin{aligned}
& \Omega_{1}=\left\{x \in \mathbb{R}^{n} \mid \sum_{i=1}^{n} \frac{x_{i}^{2}}{2} \leq \frac{\sum_{i=1}^{n}\left(M_{i}^{(1)}\right)^{2} / \varepsilon_{i}}{2 \min _{1 \leq i \leq n}\left(\underline{d_{i}}-\varepsilon_{i}\right)}, 0<\varepsilon_{i}<\underline{d_{i}}\right\}, \\
& \Omega_{2}=\left\{x \in \mathbb{R}^{n}|| x_{i} \mid \leq 2 M_{i}^{(1)} / \underline{d_{i}}\right\}, \\
& \Omega_{3}=\left\{x \in \mathbb{R}^{n}\left|\sum_{i=1}^{n}\right| x_{i} \mid \leq \frac{\sum_{i=1}^{n} 2 M_{i}^{(1)}}{\min _{1 \leq i \leq n} \underline{d_{i}}}\right\} .
\end{aligned}
$$

Proof. We prove the uniform stability of (2.1) in Lagrange sense by considering different Lyapunov functions.

- First, we employ the radially unbounded and positive definite Lyapunov function

$$
V(x(t))=\frac{1}{2} \sum_{i=1}^{n} x_{i}^{2}(t) .
$$


Computing the derivative of $V(x(t))$ along the positive half trajectory of (2.1), we obtain

$$
\begin{aligned}
\left.D^{+} V(x)(t)\right|_{[2.1]}= & \sum_{i=1}^{n} x_{i}(t) \frac{d x_{i}(t)}{d t} \\
= & \sum_{i=1}^{n} x_{i}(t)\left[-d_{i}(t) x_{i}(t)+\sum_{j=1}^{n} a_{i j}(t) f_{j}\left(x_{j}(t)\right)\right. \\
& \left.+\sum_{j=1}^{n} b_{i j}(t) g_{j}\left(x_{j}\left(t-\tau_{i j}(t)\right)\right)+I_{i}(t)\right] \\
\leq & \left.\sum_{i=1}^{n}-\underline{d_{i}} x_{i}^{2}(t)+\sum_{i=1}^{n}\left(\sum_{j=1}^{n}\left|\bar{a}_{i j}\right| h_{j}+\sum_{j=1}^{n}\left|\bar{b}_{i j}\right| k_{j}\right)+\left|\bar{I}_{i}\right|\right)\left|x_{i}(t)\right| \\
\leq & -\sum_{i=1}^{n} \underline{d_{i}} x_{i}^{2}(t)+\sum_{i=1}^{n} 2 M_{i}^{(1)}\left|x_{i}(t)\right| \\
\leq & -\sum_{i=1}^{n} \underline{d_{i}} x_{i}^{2}(t)+\sum_{i=1}^{n} \varepsilon_{i} x_{i}^{2}(t)+\sum_{i=1}^{n} \frac{\left(M_{i}^{(1)}\right)^{2}}{\varepsilon_{i}} \\
\leq & -\min _{1 \leq i \leq n}\left\{\underline{d_{i}}-\varepsilon_{i}\right\} \sum_{i=1}^{n} x_{i}^{2}(t)+\sum_{i=1}^{n} \frac{\left(M_{i}^{(1)}\right)^{2}}{\varepsilon_{i}} \\
\leq & -2 \min _{1 \leq i \leq n}\left\{\underline{d_{i}}-\varepsilon_{i}\right\} V(x)(t)+\sum_{i=1}^{n} \frac{\left(M_{i}^{(1)}\right)^{2}}{\varepsilon_{i}} \\
= & -\alpha V(x(t))+\beta,
\end{aligned}
$$

where $\alpha=2 \min _{1 \leq i \leq n}\left\{\underline{d_{i}}-\varepsilon_{i}\right\}$ and $\beta=\sum_{i=1}^{n}\left(M_{i}^{(1)}\right)^{2} / \varepsilon_{i}$. By Lemma 2.6. for $t \geq t_{0}$ we have

$$
V(x(t))-\frac{\beta}{\alpha} \leq\left(V\left(x\left(t_{0}\right)\right)-\frac{\beta}{\alpha}\right) e^{-\alpha\left(t-t_{0}\right)} .
$$

This implies that the solutions of (2.1) are uniformly bounded. Hence (2.1) is uniformly stable in Lagrange sense. Also

$$
V(x(0))-\frac{\beta}{\alpha} \leq V(x(0))=\frac{1}{2} \sum_{i=1}^{n} x_{i}^{2}(0)=\frac{1}{2} \sum_{i=1}^{n} \phi_{i}^{2}(0)=K(\phi) .
$$

Then $K \in \mathcal{C}_{\mathcal{F}}^{+}$and from 3.3 , we can rewrite 3.4 as

$$
V(x(t))-\frac{\beta}{\alpha} \leq K(\phi) e^{-\alpha t}, \quad t \geq 0 .
$$

By Definition 2.3, network (2.1) is globally exponentially stable and $\Omega_{1}$ is a globally exponentially attractive set. 
- To prove that $\Omega_{2}$ is GEA, we take the $n$ radially unbounded and positive definite Lyapunov functions

$$
V_{i}\left(x_{i}(t)\right)=\left|x_{i}(t)\right|, \quad i=1, \ldots, n .
$$

Then on differentiation we obtain

$$
\begin{aligned}
\left.D^{+} V_{i}\left(x_{i}\right)(t)\right|_{\underline{2.1}} & \leq-\underline{d_{i}}\left|x_{i}(t)\right|+\sum_{j=1}^{n}\left|\bar{a}_{i j}\right| h_{j}+\sum_{j=1}^{n}\left|\bar{b}_{i j}\right| k_{j}+\left|\bar{I}_{i}\right| \\
& =-\underline{d_{i}} V_{i}\left(x_{i}(t)\right)+2 M_{i}^{(1)} .
\end{aligned}
$$

Then according to Lemma 2.6 .

$$
V_{i}\left(x_{i}(t)\right)-\frac{2 M_{i}^{(1)}}{\underline{d_{i}}} \leq\left(V_{i}\left(x_{i}\left(t_{0}\right)\right)-\frac{2 M_{i}^{(1)}}{\underline{d_{i}}}\right) e^{-\underline{d_{i}}\left(t-t_{0}\right)} .
$$

Let $K_{i}(\phi)=V_{i}\left(\phi_{i}(0)\right)=\left|\phi_{i}(0)\right|, i=1, \ldots, n$. Then

$$
V_{i}\left(x_{i}(t)\right)-\frac{2 M_{i}^{(1)}}{\underline{d_{i}}} \leq K_{i}(\phi) e^{-\alpha t},
$$

where $\alpha=\underline{d_{i}}$. Thus, we conclude that $K_{i} \in \mathcal{C}_{\mathcal{F}}^{+}$and so $\Omega_{2}$ is a GEA set.

- Next, we consider the radially unbounded and positive definite Lyapunov function

$$
V(x(t))=\sum_{i=1}^{n}\left|x_{i}(t)\right| .
$$

Computing the upper right derivative, we obtain

$$
\begin{aligned}
\left.D^{+} V(x(t))\right|_{\underline{2.1}} & \leq-\sum_{i=1}^{n}\left(\underline{d_{i}}\left|x_{i}(t)\right|+\sum_{j=1}^{n}\left(\left|\bar{a}_{i j}\right| h_{j}+\left|\bar{b}_{i j}\right| k_{j}\right)+\left|\bar{I}_{i}\right|\right) \\
& =-\sum_{i=1}^{n} \underline{d_{i}}\left|x_{i}(t)\right|+\sum_{i=1}^{n} 2 M_{i}^{(1)} \\
& \leq-\min _{1 \leq i \leq n} \frac{d_{i}}{} \cdot \sum_{i=1}^{n}\left|x_{i}(t)\right|+\sum_{i=1}^{n} 2 M_{i}^{(1)} \\
& =-\alpha V(x(t))+\beta
\end{aligned}
$$

where $\alpha=\min _{1 \leq i \leq n} \underline{d_{i}}$ and $\beta=\sum_{i=1}^{n} 2 M_{i}^{(1)}$. Again in the same manner, applying Lemma 2.6 for $t \geq t_{0}$ we get

$$
V(x(t))-\frac{\beta}{\alpha} \leq\left(V\left(x\left(t_{0}\right)\right)-\frac{\beta}{\alpha}\right) e^{-\alpha\left(t-t_{0}\right)} .
$$


Now from Lemma 2.6 and Definition 2.3 .

$$
V(x(t))-\frac{\sum_{i=1}^{n} 2 M_{i}^{(1)}}{\min _{1 \leq i \leq n} \underline{d_{i}}} \leq\left(V(x(0))-\frac{\sum_{i=1}^{n} 2 M_{i}^{(1)}}{\min _{1 \leq i \leq n} \underline{d_{i}}}\right) e^{-\alpha t}, \quad t \geq 0 .
$$

Let $K(\phi)=V(x(0))=V(\phi(0))=\sum_{i=1}^{n}\left|\phi_{i}(0)\right|$. Then $K \in \mathcal{C}_{\mathcal{F}}^{+}$and hence

$$
V(x(t))-\frac{\sum_{i=1}^{n} 2 M_{i}^{(1)}}{\min _{1 \leq i \leq n} \underline{d_{i}}} \leq K(\phi) e^{-\alpha t}, \quad t \geq 0 .
$$

Hence by Definition $2.3, \Omega_{3}$ is a GEA set.

REMARK 3.2. Since $\Omega_{1}, \Omega_{2}, \Omega_{3}$ are GEA sets of the network (2.1), we see that $\Omega_{123}=\Omega_{1} \cap \Omega_{2} \cap \Omega_{3}$ is a better GEA set of (2.1).

Corollary 3.3. If $f_{i} \in \mathcal{B}$, then the network (2.3) is globally exponentially stable in Lagrange sense with GEA sets

$$
\begin{aligned}
& \Omega_{4}=\left\{x \in \mathbb{R}^{n} \mid \sum_{i=1}^{n} \frac{x_{i}^{2}}{2} \leq \frac{\sum_{i=1}^{n}\left(M_{i}^{(2)}\right)^{2} / \varepsilon_{i}}{2 \min _{1 \leq i \leq n}\left(\underline{d_{i}}-\varepsilon_{i}\right)}, 0<\varepsilon_{i}<\underline{d_{i}}\right\}, \\
& \Omega_{5}=\left\{x \in \mathbb{R}^{n}|| x_{i} \mid \leq 2 M_{i}^{(2)} / \underline{d_{i}}\right\}, \\
& \Omega_{6}=\left\{x \in \mathbb{R}^{n}\left|\sum_{i=1}^{n}\right| x_{i} \mid \leq \frac{\sum_{i=1}^{n} 2 M_{i}^{(2)}}{\min _{1 \leq i \leq n} \underline{d_{i}}}\right\} .
\end{aligned}
$$

Hence $\Omega_{456}=\Omega_{4} \cap \Omega_{5} \cap \Omega_{6}$ is another globally exponentially attractive set of (2.3).

Corollary 3.4. If $g_{i}(\cdot) \in \mathcal{B}$, then the network 2.4 is globally exponentially stable in Lagrange sense with GEA sets of

$$
\begin{aligned}
& \Omega_{7}=\left\{x \in \mathbb{R}^{n} \mid \sum_{i=1}^{n} \frac{x_{i}^{2}}{2} \leq \frac{\sum_{i=1}^{n}\left(M_{i}^{(3)}\right)^{2} / \varepsilon_{i}}{2 \min _{1 \leq i \leq n}\left(\underline{d_{i}}-\varepsilon_{i}\right)}, 0<\varepsilon_{i}<\underline{d_{i}}\right\}, \\
& \Omega_{8}=\left\{x \in \mathbb{R}^{n}|| x_{i} \mid \leq 2 M_{i}^{(3)} / \underline{d_{i}}\right\}, \\
& \Omega_{9}=\left\{x \in \mathbb{R}^{n}\left|\sum_{i=1}^{n}\right| x_{i} \mid \leq \frac{\sum_{i=1}^{n} 2 M_{i}^{(3)}}{\min _{1 \leq i \leq n} \underline{d_{i}}}\right\} .
\end{aligned}
$$

Hence $\Omega_{789}=\Omega_{7} \cap \Omega_{8} \cap \Omega_{9}$ is another GEA set of (2.4).

REMARK 3.5. The globally exponentially attractive sets $\Omega_{i}(i=1, \ldots, n)$ derived in Theorem 3.1 may not be optimal. One can choose other attractive sets also.

3.2. Sigmoidal and bounded activation functions. In this subsection, we assume that $f_{i} \in \mathcal{S}$ and $g_{i} \in \mathcal{B}$. Consider the matrix form of the network 2.1):

$$
\dot{X}(t)=-D(t) X(t)+A(t) F(X(t))+B(t) G(X(t-\tau(t)))+I(t) .
$$


For $B(t)=0$, the network 3.9 reduces to

$$
\dot{X}(t)=-D(t) X(t)+A(t) F(X(t))+I(t),
$$

where $D(t)=\operatorname{diag}\left\{d_{1}(t), \ldots, d_{n}(t)\right\}, d_{i}(t) \in \mathbb{R}^{+}, A(t)=\left(a_{i j}(t)\right)_{n \times n}, B(t)=$ $\left(b_{i j}(t)\right)_{n \times n}, I(t)=\left(I_{1}(t), \ldots, I_{n}(t)\right)^{T}$ and $\tau(t)=\left(\tau_{i j}(t)\right)_{n \times n}$. Let $h_{i}, k_{i}>0$ be the saturation constants of $f_{i}$ and $g_{i}, i=1, \ldots, n$. For $l=1,2,3$, choose matrices $P^{(l)}=\left(p_{i j}^{(l)}\right)_{n \times n}$ and $Q^{(l)}=\left(q_{i j}^{(l)}\right)_{n \times n}$ such that $\bar{A}^{(l)}=A+P^{(l)}=$ $\left(\bar{a}_{i j}^{(l)}\right)_{n \times n}$ and $\bar{B}^{(l)}=B+Q^{(l)}=\left(\bar{b}_{i j}^{(l)}\right)_{n \times n}$ satisfy

- $\bar{A}^{(1)}+\left(\bar{A}^{(1)}\right)^{T}<0$ and $\bar{B}^{(1)}+\left(\bar{B}^{(1)}\right)^{T}<0$ are negative definite,

- $\bar{A}^{(2)}+\left(\bar{A}^{(2)}\right)^{T} \leq 0$ and $\bar{B}^{(2)}+\left(\bar{B}^{(2)}\right)^{T} \leq 0$ are negative semi-definite,

- $a_{i j}^{(3)}+\sum_{i=1, i \neq j}^{n}\left|\bar{a}_{i j}^{(3)}\right| \leq 0$ and $b_{i j}^{(3)}+\sum_{i=1, i \neq j}^{n}\left|\bar{b}_{i j}^{(3)}\right| \leq 0$.

Choose the following constants:

$$
\begin{aligned}
\bar{M}_{i} & =\frac{1}{2} \sum_{j=1}^{n}\left(\left|p_{i j}^{(1)}\right| h_{j}+\left|\bar{b}_{i j}\right| k_{j}+\left|\bar{I}_{i}\right|\right), \\
\bar{N}_{i} & =\frac{1}{2} \sum_{j=1}^{n}\left(\left|\bar{a}_{i j}\right| h_{j}+\left|q_{i j}^{(1)}\right| k_{j}+\left|\bar{I}_{i}\right|\right), \\
\bar{M} & =\sum_{i=1}^{n}\left[\sum_{j=1}^{n}\left|p_{i j}^{(2)}\right| h_{j}+\sum_{j=1}^{n}\left|\bar{b}_{i j}\right| k_{j}+\left|\bar{I}_{i}\right|\right] h_{i}, \\
\overline{\bar{N}} & =\sum_{i=1}^{n}\left[\sum_{j=1}^{n}\left|\bar{a}_{i j}\right| h_{j}+\sum_{j=1}^{n}\left|q_{i j}^{(2)}\right| k_{j}+\left|\bar{I}_{i}\right|\right] k_{i}, \\
M^{\prime} & =\sum_{i=1}^{n}\left[\sum_{j=1}^{n}\left(\left|p_{i j}^{(3)}\right| h_{j}+\left|\bar{b}_{i j}\right| k_{j}+\left|\bar{I}_{i}\right|\right)\right], \\
M^{\prime \prime} & =\sum_{i=1}^{n}\left[\sum_{j=1}^{n}\left(\left|p_{i j}^{(3)}\right| h_{j}+\left|\bar{I}_{i}\right|\right)\right] .
\end{aligned}
$$

Theorem 3.6. Assume that $f_{i} \in \mathcal{S}$ and $g_{i} \in \mathcal{B}$. Then the PNN (3.9) is globally exponentially stable in Lagrange sense with globally exponentially attractive sets

$$
\begin{aligned}
& \Omega_{10}=\left\{x \in \mathbb{R}^{n} \mid \sum_{i=1}^{n} \int_{0}^{x_{i}(t)}\left(f_{i}(y)+g_{i}(y)\right) d y \leq \frac{\sum_{i=1}^{n}\left(\bar{M}_{i}^{2}+\bar{N}_{i}^{2}\right) / \epsilon}{\min _{1 \leq i \leq n} \underline{d_{i}}}\right\}, \\
& \Omega_{11}=\left\{x \in \mathbb{R}^{n} \mid \sum_{i=1}^{n} \int_{0}^{x_{i}(t)}\left(f_{i}(y)+g_{i}(y)\right) d y \leq \frac{\bar{M}+\overline{\bar{N}}}{\min _{1 \leq i \leq n} \underline{d_{i}}}\right\}, \\
& \Omega_{12}=\left\{x \in \mathbb{R}^{n}\left|\sum_{i=1}^{n}\right| x_{i} \mid \leq \frac{M^{\prime}}{\min _{1 \leq i \leq n} \underline{d_{i}}}\right\},
\end{aligned}
$$


where $0<\epsilon_{1}, \epsilon_{2}<\epsilon \ll 1, \bar{A}+\bar{A}^{T}+\epsilon_{1} I_{n} \leq 0$ and $\bar{B}+\bar{B}^{T} \leq 0$. Hence $\Omega=\Omega_{10} \cap \Omega_{11} \cap \Omega_{12}$ is another GEA set of (3.9).

Proof. We will employ three Lyapunov functions.

- Consider the Lyapunov function

$$
V(x(t))=\sum_{i=1}^{n} \int_{0}^{x_{i}(t)} f_{i}(y) d y+\sum_{i=1}^{n} \int_{0}^{x_{i}(t)} g_{i}(y) d y .
$$

On differentiating with respect to $t$, we obtain

$$
\begin{aligned}
D^{+} V(x(t))= & \sum_{i=1}^{n} f_{i}\left(x_{i}(t)\right) \dot{x}_{i}(t)+\sum_{i=1}^{n} g_{i}\left(x_{i}(t)\right) \dot{x}_{i}(t), \\
= & \sum_{i=1}^{n}\left[-d_{i}(t) x_{i}(t) f_{i}\left(x_{i}(t)\right)+\sum_{j=1}^{n} a_{i j}(t) f_{j}\left(x_{j}(t)\right) f_{i}\left(x_{i}(t)\right.\right. \\
& \left.+\sum_{j=1}^{n} b_{i j}(t) g_{j}\left(x_{j}\left(t-\tau_{i j}(t)\right)\right) f_{i}\left(x_{i}(t)\right)+I_{i}(t) f_{i}\left(x_{i}(t)\right)\right] \\
& +\sum_{i=1}^{n}\left[-d_{i}(t) x_{i}(t) g_{i}\left(x_{i}(t)\right)+\sum_{j=1}^{n} a_{i j}(t) f_{j}\left(x_{j}(t)\right) g_{i}\left(x_{i}(t)\right)\right. \\
& \left.+\sum_{j=1}^{n} b_{i j}(t) g_{j}\left(x_{j}\left(t-\tau_{i j}(t)\right)\right) g_{i}\left(x_{i}(t)\right)+I_{i}(t) g_{i}\left(x_{i}(t)\right)\right] \\
\leq & \sum_{i=1}^{n}\left[-\underline{d}_{i} x_{i}(t) f_{i}\left(x_{i}(t)\right)+\sum_{j=1}^{n} \bar{a}_{i j} f_{j}\left(x_{j}(t)\right) f_{i}\left(x_{i}(t)\right.\right. \\
& \left.+\sum_{j=1}^{n} \bar{b}_{i j} g_{j}\left(x_{j}\left(t-\tau_{i j}(t)\right)\right) f_{i}\left(x_{i}(t)\right)+\bar{I}_{i} f_{i}\left(x_{i}(t)\right)\right] \\
& +\sum_{i=1}^{n}\left[-\underline{d_{i}} x_{i}(t) g_{i}\left(x_{i}(t)\right)+\sum_{j=1}^{n} \bar{a}_{i j} f_{j}\left(x_{j}(t)\right) g_{i}\left(x_{i}(t)\right)\right. \\
& \left.+\sum_{j=1}^{n} \bar{b}_{i j} g_{j}\left(x_{j}\left(t-\tau_{i j}(t)\right)\right) g_{i}\left(x_{i}(t)\right)+\bar{I}_{i} g_{i}\left(x_{i}(t)\right)\right] \\
\leq & \sum_{i=1}^{n}\left[-\underline{d}_{i} x_{i}(t) f_{j}\left(x_{j}(t)\right) f_{i}\left(x_{i}(t)\right]\right. \\
& +\sum_{j=1}^{n} \bar{b}_{i j} g_{j}\left(x_{j}\left(t-\tau_{i j}(t)\right)\right) f_{i}\left(x_{i}(t)\right)+\bar{I}_{i} f_{i}\left(x_{i}(t)\right) \\
& (1) \\
i j & f_{j}\left(x_{j}(t)\right) f_{i}\left(x_{i}(t)\right. \\
& \\
& \\
&
\end{aligned}
$$




$$
\begin{aligned}
& +\sum_{i=1}^{n}\left[-\underline{d}_{i} x_{i}(t) g_{i}\left(x_{i}(t)\right)+\sum_{j=1}^{n} \bar{a}_{i j} f_{j}\left(x_{j}(t)\right) g_{i}\left(x_{i}(t)\right)\right. \\
& +\sum_{j=1}^{n}\left(\bar{b}_{i j}+q_{i j}^{(1)}\right) g_{j}\left(x_{j}\left(t-\tau_{i j}(t)\right)\right) g_{i}\left(x_{i}(t)\right)+\bar{I}_{i} g_{i}\left(x_{i}(t)\right) \\
& -\sum_{j=1}^{n} q_{i j}^{(1)} g_{j}\left(x_{j}\left(t-\tau_{i j}(t)\right)\right) g_{i}\left(x_{i}(t)\right] \\
& \leq-\sum_{i=1}^{n} \underline{d_{i}} x_{i}(t)\left(f_{i}\left(x_{i}(t)\right)+g_{i}\left(x_{i}(t)\right)\right) \\
& +\frac{1}{2} f^{T}(x(t))\left(\bar{A}^{(1)}+\left(\bar{A}^{(1)}\right)^{T}\right) f(x(t)) \\
& +\sum_{i=1}^{n}\left[\sum_{j=1}^{n}\left|p_{i j}^{(1)}\right| h_{j}+\sum_{j=1}^{n}\left|\bar{b}_{i j}\right| h_{j}+\left|\bar{I}_{i}\right|\right] f_{i}\left(x_{i}(t)\right) \\
& +\frac{1}{2} g^{T}(x(t))\left(\bar{B}^{(1)}+\left(\bar{B}^{(1)}\right)^{T}\right) g(x(t-\tau)) \\
& +\sum_{i=1}^{n}\left[\sum_{j=1}^{n}\left|\bar{a}_{i j}\right| h_{j}+\sum_{j=1}^{n}\left|q_{i j}^{(1)}\right| k_{j}+\left|\bar{I}_{i}\right|\right] g_{i}\left(x_{i}(t)\right) \\
& \leq-\min _{1 \leq i \leq n} \frac{d_{i}}{} \cdot\left[\sum_{i=1}^{n} \int_{0}^{x_{i}(t)}\left(f_{i}(y)+g_{i}(y)\right) d y\right] \\
& +\frac{1}{2} f^{T}(x(t))\left(\bar{A}^{(1)}+\left(\bar{A}^{(1)}\right)^{T}\right) f(x(t)) \\
& +\frac{1}{2} g^{T}(x(t))\left(\bar{B}^{(1)}+\left(\bar{B}^{(1)}\right)^{T}\right) g(x(t-\tau)) \\
& +2 \sum_{i=1}^{n} \bar{M}_{i}\left|f_{i}\left(x_{i}(t)\right)\right|+2 \sum_{i=1}^{n} \bar{N}_{i}\left|g_{i}\left(x_{i}(t)\right)\right| \\
& \leq-\min _{1 \leq i \leq n} \frac{d_{i}}{} \cdot\left[\sum_{i=1}^{n} \int_{0}^{x_{i}(t)}\left(f_{i}(y)+g_{i}(y)\right) d y\right]+\frac{1}{2} f^{T}(x(t))\left(\bar{A}^{(1)}\right. \\
& \left.+\left(\bar{A}^{(1)}\right)^{T}+\epsilon_{1} I_{n}\right) f(x(t))+\frac{1}{2} g^{T}(x(t))\left(\bar{B}^{(1)}+\left(\bar{B}^{(1)}\right)^{T}\right) g(x(t-\tau)) \\
& +\frac{1}{2} g^{T}(x(t)) \epsilon_{2} g(x(t))+\sum_{i=1}^{n} \frac{\bar{M}_{i}^{2}}{\epsilon_{1}}+\sum_{i=1}^{n} \frac{\bar{N}_{i}^{2}}{\epsilon_{2}} \\
& \leq-\min _{1 \leq i \leq n} \frac{d_{i}}{} \cdot V(x(t))+\sum_{i=1}^{n} \frac{\bar{M}_{i}^{2}}{\epsilon}+\sum_{i=1}^{n} \frac{\bar{N}_{i}^{2}}{\epsilon}=-\alpha V(x(t))+\beta,
\end{aligned}
$$


where $\alpha=\min _{1 \leq i \leq n} d_{i}$ and $\beta=\sum_{i=1}^{n}\left(\bar{M}_{i}^{2}+\bar{N}_{i}^{2}\right) / \epsilon$. Hence from Lemma 2.6 . $\Omega_{10}$ is a GEA set of (3.9).

- We again consider the positive definite and radially unbounded Lyapunov function

$$
V(x(t))=\sum_{i=1}^{n} \int_{0}^{x_{i}(t)} f_{i}(y) d y+\sum_{i=1}^{n} \int_{0}^{x_{i}(t)} g_{i}(y) d y .
$$

Differentiating, we obtain

$$
\begin{aligned}
D^{+} V(x(t)) \leq & -\sum_{i=1}^{n} d_{i}(t) x_{i}(t)\left(f_{i}\left(x_{i}(t)\right)+g_{i}\left(x_{i}(t)\right)\right) \\
& +\frac{1}{2} f^{T}(x(t))\left(\bar{A}^{(2)}+\left(\bar{A}^{(2)}\right)^{T}\right) f(x(t)) \\
& +\frac{1}{2} g^{T}(x(t))\left(\bar{B}^{(2)}+\left(\bar{B}^{(2)}\right)^{T}\right) g(x(t-\tau)) \\
& +\sum_{i=1}^{n}\left[\sum_{j=1}^{n}\left|p_{i j}^{(2)}\right| h_{j}+\sum_{j=1}^{n}\left|\bar{b}_{i j}\right| k_{j}+\left|\bar{I}_{i}\right|\right] h_{i} \\
& +\sum_{i=1}^{n}\left[\sum_{j=1}^{n}\left|\bar{a}_{i j}\right| h_{j}+\sum_{j=1}^{n}\left|q_{i j}^{(2)}\right| k_{j}+\left|\bar{I}_{i}\right|\right] k_{i} \\
\leq & -\min _{1 \leq i \leq n} \underline{d_{i}} \cdot V(x(t))+(\overline{\bar{M}}+\overline{\bar{N}})=-\alpha V(x(t))+\beta,
\end{aligned}
$$

where $\alpha=\min _{1 \leq i \leq n} \underline{d_{i}}$ and $\beta=\overline{\bar{M}}+\overline{\bar{N}}, i=1,2, \ldots$ By Lemma 2.6, $\Omega_{11}$ is a GEA set of (3.9).

- Now we consider the radially unbounded and positive definite Lyapunov function

$$
V(x(t))=\sum_{i=1}^{n}\left|x_{i}(t)\right|
$$

Calculating the upper right derivative, we obtain

$$
\begin{aligned}
D^{+} V(x(t))= & \sum_{i=1}^{n} \dot{x_{i}}(t) \operatorname{sign}\left(x_{i}(t)\right)=\sum_{i=1}^{n}\left[-d_{i}(t) x_{i}(t)+\sum_{j=1}^{n} a_{i j}(t) f_{j}\left(x_{j}(t)\right)\right. \\
& \left.+\sum_{j=1}^{n} b_{i j}(t) g_{j}\left(x_{j}\left(t-\tau_{i j}(t)\right)\right)+I_{i}(t)\right] \operatorname{sign}\left(x_{i}(t)\right), \\
= & \sum_{i=1}^{n}\left[-d_{i}(t) x_{i}(t)+\sum_{j=1}^{n}\left(\bar{a}_{i j}^{(3)}-p_{i j}^{(3)}\right) f_{j}\left(x_{j}(t)\right)\right. \\
& \left.+\sum_{j=1}^{n} b_{i j}(t) g_{j}\left(x_{j}\left(t-\tau_{i j}(t)\right)\right)+I_{i}(t)\right] .
\end{aligned}
$$


Further we break $\bar{a}_{i j}^{(3)}$ as $\bar{a}_{i j}^{(3)}=\bar{a}_{j j}^{(3)}+\sum_{i, i \neq j}\left|\bar{a}_{i j}^{(3)}\right|$. This yields

$$
\begin{aligned}
D^{+} V(x(t)) \leq & -\min _{1 \leq i \leq n} \underline{d_{i}} \cdot \sum_{i=1}^{n}\left|x_{i}(t)\right|+\sum_{j=1}^{n}\left(\bar{a}_{j j}^{(3)}+\sum_{i, i \neq j}\left|\bar{a}_{i j}^{(3)}\right|\right) f_{j}\left(x_{j}(t)\right) \\
& +\sum_{j=1}^{n} b_{i j}(t) g_{j}\left(x_{j}\left(t-\tau_{i j}(t)\right)\right)+I_{i}(t)-\sum_{j=1}^{n} p_{i j}^{(3)} f_{j}\left(x_{j}(t)\right) \\
\leq & -\min _{1 \leq i \leq n} \underline{d_{i}} \cdot \sum_{i=1}^{n}\left|x_{i}(t)\right|+\sum_{j=1}^{n}\left(\bar{a}_{j j}^{(3)}+\sum_{i, i \neq j}\left|\bar{a}_{i j}^{(3)}\right|\right) f_{j}\left(x_{j}(t)\right) \\
& +\sum_{i=1}^{n}\left[\sum_{j=1}^{n}\left(\left|p_{i j}^{(3)}\right| h_{j}+\left|\bar{b}_{i j}\right| k_{j}\right)+\left|\bar{I}_{i}\right|\right] \\
\leq & -\min _{1 \leq i \leq n} \underline{d_{i}} \cdot V(x(t))+M^{\prime}=-\alpha V(x(t))+\beta,
\end{aligned}
$$

with $\alpha=\min _{1 \leq i \leq n} \underline{d_{i}}$ and $\beta=M^{\prime}$.

Again, from Lemma 2.6, $\Omega_{12}$ is a GEA set of 3.9 .

Corollary 3.7. Assume that $f_{i} \in \mathcal{S}$ and $g_{i} \in \mathcal{B}$. Then the PNN 3.10) is globally exponentially stable in Lagrange sense with globally exponentially attractive set

$$
\Omega_{13}=\left\{x \in \mathbb{R}^{n}\left|\sum_{i=1}^{n}\right| x_{i}(t) \mid \leq \frac{M^{\prime \prime}}{\min _{1 \leq i \leq n} \underline{d_{i}}}\right\} .
$$

4. Global exponential stability for $f_{i}=g_{i}$. In this subsection we consider $f_{i}=g_{i}$. In this case, the PNN under consideration takes the form

$$
\begin{aligned}
\frac{d x_{i}(t)}{d t}= & -d_{i}(t) x_{i}(t)+\sum_{j=1}^{n} a_{i j}(t) f_{j}\left(x_{j}(t)\right) \\
& +\sum_{j=1}^{n} b_{i j}(t) f_{j}\left(x_{j}\left(t-\tau_{i j}(t)\right)\right)+I_{i}(t) .
\end{aligned}
$$

The assumptions on the system are as in Section 2. The PNN 4.1) is analyzed considering bounded activation functions and sigmoidal activation functions. This network is a generalization of the periodic neural network model considered in [22] as it consists of time-varying delays.

If $b_{i j}(t)=0$, then 4.1 reduces to

$$
\frac{d x_{i}(t)}{d t}=-d_{i}(t) x_{i}(t)+\sum_{j=1}^{n} a_{i j}(t) f_{j}\left(x_{j}(t)\right)+I_{i}(t) .
$$


For $a_{i j}(t)=0,4.1$ takes the form

$$
\frac{d x_{i}(t)}{d t}=-d_{i}(t) x_{i}(t)+\sum_{j=1}^{n} b_{i j}(t) f_{j}\left(x_{j}\left(t-\tau_{i j}(t)\right)\right)+I_{i}(t) .
$$

4.1. Bounded activation functions. In this subsection, we consider the network (4.1) under the same assumptions as in Subsection 3.1 with $f_{i} \in \mathcal{B}$. Let $h_{i}>0$ be the saturation constants of $f_{i}(i=1, \ldots, n)$. Define

$$
M_{i}^{(4)}=\frac{1}{2}\left(\sum_{j=1}^{n}\left(\left|\bar{a}_{i j}\right|+\left|\bar{b}_{i j}\right|\right) h_{j}+\left|\bar{I}_{i}\right|\right), \quad M_{i}^{(5)}=\frac{1}{2}\left(\sum_{j=1}^{n}\left|\bar{b}_{i j}\right| h_{j}+\left|\bar{I}_{i}\right|\right) .
$$

Theorem 4.1. Assume that $f_{i} \in \mathcal{B}$, i.e. $\left|f_{i}\right| \leq h_{i}$ with $h_{i}>0$ and $x_{i} f_{i}\left(x_{i}\right)>0$ for $x_{i} \neq 0, f_{i}(0)=0, i=1, \ldots, n$. Then the network (4.1) is globally exponentially stable in Lagrange sense with globally exponentially attractive sets

$$
\begin{aligned}
& \Omega_{a}=\left\{x \in \mathbb{R}^{n} \mid \sum_{i=1}^{n} \frac{x_{i}^{2}(t)}{2} \leq \frac{\sum_{i=1}^{n}\left(M_{i}^{(4)}\right)^{2} / \varepsilon_{i}^{\prime}}{2 \min _{1 \leq i \leq n}\left(\underline{d_{i}}-\varepsilon_{i}^{\prime}\right)}, 0<\varepsilon_{i}^{\prime}<\underline{d_{i}}\right\}, \\
& \Omega_{b}=\left\{x \in \mathbb{R}^{n}|| x_{i}(t) \mid \leq 2 M_{i}^{(4)} / \underline{d_{i}}\right\}, \\
& \Omega_{c}=\left\{x \in \mathbb{R}^{n}\left|\sum_{i=1}^{n}\right| x_{i}(t) \mid \leq \frac{\sum_{i=1}^{n} 2 M_{i}^{(4)}}{\min _{1 \leq i \leq n} \underline{d_{i}}}\right\} .
\end{aligned}
$$

Hence $\Omega=\Omega_{a} \cap \Omega_{b} \cap \Omega_{c}$ is another GEA set of 4.1.

Proof. The proof follows similar steps to those for Theorem 3.1.

REMARK 4.2. The size of the globally exponentially attractive sets $\Omega_{1}$ and $\Omega_{a}$ is related to the exponential decay rate of trajectories. It involves selection of $\varepsilon_{i}, \varepsilon_{i}^{\prime}>0$. The smaller the $\varepsilon_{i}, \varepsilon_{i}^{\prime}$, the larger the attractive set, and hence the convergence rate of the trajectories into $\Omega_{1}$ and $\Omega_{a}$ gets greater. So selecting smaller $\varepsilon_{i}, \varepsilon_{i}^{\prime}$ is preferred.

Corollary 4.3. If $f_{i} \in \mathcal{B}$, then the network (4.3) is GES in Lagrange sense with GEA sets

$$
\begin{aligned}
& \Omega_{d}=\left\{x \in \mathbb{R}^{n} \mid \sum_{i=1}^{n} \frac{x_{i}^{2}(t)}{2} \leq \frac{\sum_{i=1}^{n}\left(M_{i}^{(5)}\right)^{2} / \varepsilon_{i}}{2 \min _{1 \leq i \leq n}\left(\underline{d_{i}}-\varepsilon_{i}^{\prime}\right)}, 0<\varepsilon_{i}^{\prime}<\underline{d_{i}}\right\}, \\
& \Omega_{e}=\left\{x \in \mathbb{R}^{n}|| x_{i}(t) \mid \leq 2 M_{i}^{(5)} / \underline{d_{i}}\right\}, \\
& \Omega_{f}=\left\{x \in \mathbb{R}^{n}\left|\sum_{i=1}^{n}\right| x_{i}(t) \mid \leq \frac{\sum_{i=1}^{n} 2 M_{i}^{(5)}}{\min _{1 \leq i \leq n} \underline{d_{i}}}\right\} .
\end{aligned}
$$

Hence $\Omega=\Omega_{d} \cap \Omega_{e} \cap \Omega_{f}$ is another GEA set of (4.3). 
REMARK 4.4. For $b_{i j}(t)=0$, the network 4.2 takes the form of 2.3 . This case has already been discussed in Corollary 3.3 .

4.2. Sigmoidal activation functions. In this subsection, we consider the network (4.1) with $f_{i} \in \mathcal{S}$. Let $m_{j}(j=1, \ldots, n)$ be the saturation constant of $f_{j}(\cdot)$. Under similar notations to those in Subsection 3.2, consider the matrix form of network 4.1),

$$
\dot{X}(t)=-D(t) X(t)+A(t) F(X(t))+B(t) F(X(t-\tau(t)))+I(t) .
$$

For $B(t)=0$, the network 3.9 reduces to

$$
\dot{X}(t)=-D(t) X(t)+A(t) F(X(t))+I(t) .
$$

Choose the following constants:

$$
\begin{aligned}
& H_{i}^{(1)}=\frac{1}{2}\left(\sum_{j=1}^{n}\left(\left|p_{i j}^{(1)}\right|+\left|\bar{b}_{i j}\right|\right) m_{j}+\left|\bar{I}_{i}\right|\right), \\
& H^{(2)}=\sum_{i=1}^{n}\left(\sum_{j=1}^{n}\left(\left|p_{i j}^{(2)}\right|+\left|\bar{b}_{i j}\right|\right) m_{j}+\left|\bar{I}_{i}\right|\right) m_{i}, \\
& \left.H^{(3)}=\sum_{i=1}^{n}\left(\sum_{j=1}^{n}\left(\left|p_{i j}^{(3)}\right|+\left|\bar{b}_{i j}\right|\right) m_{j}+\left|\bar{I}_{i}\right|\right)\right), \\
& H^{(4)}=\sum_{i=1}^{n}\left(\sum_{j=1}^{n}\left|p_{i j}^{(3)}\right| m_{j}+\left|\bar{I}_{i}\right|\right) .
\end{aligned}
$$

Theorem 4.5. Assume that $f_{i} \in \mathcal{S}$. Then PNN (4.5) is globally exponentially stable in Lagrange sense with globally exponentially attractive sets

$$
\begin{aligned}
\Omega_{m} & =\left\{x \in \mathbb{R}^{n} \mid \sum_{i=1}^{n} \int_{0}^{x_{i}(t)} f_{i}(y) d y \leq \frac{\sum_{i=1}^{n}\left(H_{i}^{(1)}\right)^{2} / \epsilon^{\prime \prime}}{\min _{1 \leq i \leq n} \underline{d_{i}}}\right\}, \\
\Omega_{n} & =\left\{x \in \mathbb{R}^{n} \mid \sum_{i=1}^{n} \int_{0}^{x_{i}(t)} f_{i}(y) d y \leq \frac{H^{(2)}}{\min _{1 \leq i \leq n} \underline{d_{i}}}\right\}, \\
\Omega_{p} & =\left\{x \in \mathbb{R}^{n}\left|\sum_{i=1}^{n}\right| x_{i}(t) \mid \leq \frac{H^{(3)}}{\min _{1 \leq i \leq n} \underline{d_{i}}}\right\},
\end{aligned}
$$

where $0<\epsilon^{\prime \prime} \ll 1, \bar{A}+\bar{A}^{T}+\epsilon^{\prime \prime} I_{n} \leq 0$ and $\bar{B}+\bar{B}^{T} \leq 0$. Hence $\Omega=\Omega_{m} \cap \Omega_{n} \cap \Omega_{p}$ is another GEA set of 4.5).

Proof. We employ three Lyapunov functions.

- Consider the Lyapunov function

$$
V(x(t))=\sum_{i=1}^{n} \int_{0}^{x_{i}(t)} f_{i}(y) d y .
$$


On differentiating with respect to $t$, we get

$$
\begin{aligned}
& \left.D^{+} V(x(t))\right|_{4.5}=\sum_{i=1}^{n} f_{i}\left(x_{i}(t)\right) \dot{x}_{i}(t) \\
& \leq \sum_{i=1}^{n}\left[-\underline{d}_{i} x_{i}(t) f_{i}\left(x_{i}(t)\right)+\sum_{j=1}^{n} \bar{a}_{i j} f_{j}\left(x_{j}(t)\right) f_{i}\left(x_{i}(t)\right)\right. \\
& \left.+\sum_{j=1}^{n} \bar{b}_{i j} f_{j}\left(x_{j}\left(t-\tau_{i j}(t)\right)\right) f_{i}\left(x_{i}(t)\right)+\bar{I}_{i} f_{i}\left(x_{i}(t)\right)\right] \\
& \leq \sum_{i=1}^{n}\left[-\underline{d}_{i} x_{i}(t) f_{i}\left(x_{i}(t)\right)+\sum_{j=1}^{n}\left(\bar{a}_{i j}+p_{i j}^{(1)}\right) f_{j}\left(x_{j}(t)\right) f_{i}\left(x_{i}(t)\right)\right. \\
& +\sum_{j=1}^{n} \bar{b}_{i j} f_{j}\left(x_{j}\left(t-\tau_{i j}(t)\right)\right) f_{i}\left(x_{i}(t)\right)+\bar{I}_{i} f_{i}\left(x_{i}(t)\right) \\
& \left.-p_{i j}^{(1)} f_{j}\left(x_{j}(t)\right) f_{i}\left(x_{i}(t)\right)\right] \\
& \leq \sum_{i=1}^{n}-\underline{d_{i}} x_{i}(t) f_{i}\left(x_{i}(t)\right)+\frac{1}{2} f^{T}(x(t))\left[\bar{A}^{(1)}+\left(\bar{A}^{(1)}\right)^{T}\right] f(x(t)) \\
& +\sum_{i=1}^{n}\left[\sum_{j=1}^{n}\left(\left|p_{i j}^{(1)}\right| m_{j}+\left|\bar{b}_{i j}\right| m_{j}\right)+\left|\bar{I}_{i}\right|\right]\left|f_{i}\left(x_{i}(t)\right)\right| f(x(t)) \\
& +2 \sum_{i=1}^{n} H_{i}^{(1)}\left|f_{i}\left(x_{i}(t)\right)\right| \\
& \leq-\min _{1 \leq i \leq n} \underline{d_{i}} \cdot\left[\sum_{i=1}^{n} \int_{0}^{x_{i}(t)} f_{i}(y) d y\right] \\
& +\frac{1}{2} f^{T}(x(t))\left[\bar{A}^{(1)}+\left(\bar{A}^{(1)}\right)^{T}+\epsilon^{\prime \prime} I_{n}\right] f(x(t)) \sum_{i=1}^{n} \frac{\left(H_{i}^{(1)}\right)^{2}}{\epsilon^{\prime \prime}} \\
& \leq-\min _{1 \leq i \leq n} \frac{d_{i}}{} \cdot V(x(t))+\sum_{i=1}^{n} \frac{\left(H_{i}^{(1)}\right)^{2}}{\epsilon^{\prime \prime}}=-\alpha V(x(t))+\beta,
\end{aligned}
$$

where $\alpha=\min _{1 \leq i \leq n} \underline{d_{i}}$ and $\beta=\sum_{i=1}^{n}\left(H_{i}^{(1)}\right)^{2} / \epsilon^{\prime \prime}$. If

$$
V\left(x\left(t_{0}\right)\right)>\frac{\beta}{\alpha}, \quad V(x(t))>\frac{\beta}{\alpha}, t \geq t_{0},
$$

then using Lemma 2.6 we obtain

$$
V(x(t))-\frac{\beta}{\alpha} \leq\left(V\left(x\left(t_{0}\right)\right)-\frac{\beta}{\alpha}\right) e^{-\alpha\left(t-t_{0}\right)} .
$$

Hence $\Omega_{m}$ is a GEA set of 4.5 . 
- We employ the same positive definite and radially unbounded Lyapunov function as in (4.7):

$$
V(x(t))=\sum_{i=1}^{n} \int_{0}^{x_{i}(t)} f_{i}(y) d y .
$$

On differentiation, we obtain

$$
\begin{aligned}
\left.D^{+} V(x(t))\right|_{[4.5)} \leq & -\sum_{i=1}^{n} \frac{d_{i}}{x_{i}}(t) f_{i}\left(x_{i}(t)\right)+\frac{1}{2} f^{T}(x(t))\left[\bar{A}^{(2)}+\left(\bar{A}^{(2)}\right)^{T}\right] f(x(t)) \\
& +\sum_{i=1}^{n}\left[\sum_{j=1}^{n}\left(\left|p_{i j}^{(2)}\right| m_{j}+\left|\bar{b}_{i j}\right| m_{j}\right)+\left|\bar{I}_{i}\right|\right] m_{i} \\
\leq & -\min _{1 \leq i \leq n} \frac{d_{i}}{} \cdot V(x(t))+H^{(2)}=-\alpha V(x(t))+\beta,
\end{aligned}
$$

where $\alpha=\min _{1 \leq i \leq n} d_{i}$ and $\beta=H^{(2)}$. Using Lemma 2.6, we conclude that $\Omega_{n}$ is a GEA set of 4.5 .

- Now we consider the radially unbounded and positive definite Lyapunov function

$$
V(x(t))=\sum_{i=1}^{n}\left|x_{i}(t)\right| .
$$

Then

$$
\begin{aligned}
\left.D^{+} V(x(t))\right|_{[4.5] \leq} & \sum_{i=1}^{n}\left[-\underline{d_{i}} x_{i}(t)+\sum_{j=1}^{n} \bar{a}_{i j} f_{j}\left(x_{j}(t)\right)\right. \\
& \left.+\sum_{j=1}^{n} \bar{b}_{i j} f_{j}\left(x_{j}\left(t-\tau_{i j}(t)\right)\right)+\bar{I}_{i}\right] \operatorname{sign}\left(x_{i}(t)\right) \\
\leq & \sum_{i=1}^{n}\left[-\underline{d_{i}} x_{i}(t)+\sum_{j=1}^{n} \bar{a}_{i j}^{(3)} f_{j}\left(x_{j}(t)\right)-\sum_{j=1}^{n} \bar{p}_{i j}^{(3)} f_{j}\left(x_{j}(t)\right)\right. \\
& \left.+\sum_{j=1}^{n} \bar{b}_{i j} f_{j}\left(x_{j}\left(t-\tau_{i j}(t)\right)\right)+\bar{I}_{i}\right] \operatorname{sign}\left(x_{i}(t)\right) \\
\leq & -\sum_{i=1}^{n} \underline{d_{i}}\left|x_{i}(t)\right|+\sum_{j=1}^{n}\left(\bar{a}_{j j}^{(3)}+\sum_{i=1, i \neq j}^{n}\left|\bar{a}_{i j}^{(3)}\right|\right)\left|f_{j}\left(x_{j}(t)\right)\right| \\
& +\sum_{i=1}^{n}\left[\sum_{j=1}^{n}\left(\left|p_{i j}^{(3)}\right|+\left|\bar{b}_{i j}\right|\right) m_{j}+\bar{I}_{i}\right] \\
\leq & -\min _{1 \leq i \leq n} \underline{d_{i}} \cdot V(x(t))+H^{(3)} .
\end{aligned}
$$


From Lemma 2.6, for $t \geq t_{0}$, we have

$$
V(x(t))-\frac{H^{(3)}}{\min _{1 \leq i \leq n} \underline{d_{i}}} \leq\left(V\left(x\left(t_{0}\right)\right)-\frac{H^{(3)}}{\min _{1 \leq i \leq n} \underline{d_{i}}}\right) e^{-\min _{1 \leq i \leq n} \underline{d_{i}\left(t-t_{0}\right)} .}
$$

Hence from Lemma 2.6. $\Omega_{p}$ is a GEA set of 4.5).

Corollary 4.6. Assume that $f_{i} \in \mathcal{S}$. Then PNN (4.6) is GES in Lagrange sense with globally exponentially stable set

$$
\Omega_{q}=\left\{x \in \mathbb{R}^{n}\left|\sum_{i=1}^{n}\right| x_{i}(t) \mid \leq \frac{H^{(4)}}{\min _{1 \leq i \leq n} \underline{d_{i}}}\right\} .
$$

Remark 4.7. According to Theorems 3.1 4.5, outside a globally exponentially attractive set, there is no equilibrium point, periodic solution, almost periodic solution or chaos attractor of the neural network.

REMARK 4.8. In 22 the neural network model considered consists of a single activation function, whereas in this work we have considered periodic neural networks (2.1) consisting of different activation functions and multiple time-varying delays. Thus the results derived are more general and can be applied to any general neural network model with several activation functions.

\section{Numerical example}

EXAmPle 5.1. Consider the neural network model

$$
\dot{x}(t)=-d(t) x(t)+A F(x(t))+B G(x(t-\tau)),
$$

where $F(x(t))=\left(f\left(x_{1}(t)\right), f\left(x_{2}(t)\right), f\left(x_{3}(t)\right)\right)^{T}, G(x(t-\tau))=\left(g\left(x_{1}(t-\tau)\right)\right.$, $\left.g\left(x_{2}(t-\tau)\right), g\left(x_{3}(t-\tau)\right)\right)^{T}$, with bounded activation functions $f(x)=g(x)=$ $(|x+1|-|x-1|) / 2$, and

$$
A=\left(\begin{array}{ccc}
1.5 & -1.4 & 0 \\
1.5 & 1+s & 0.8 \\
0 & 1.2 & 1.6
\end{array}\right), \quad B=\left(\begin{array}{ccc}
1.2 & -0.8 & 0 \\
1.4 & 1.2 & 1+s \\
1.2 & 0 & 1.2+s
\end{array}\right), \quad d=\left(\begin{array}{lll}
1 & 0 & 0 \\
0 & 1 & 0 \\
0 & 0 & 1
\end{array}\right) \text {. }
$$

For $s=-0.8,2 M_{1}=\sum_{j=1}^{3}\left(\left|a_{1 j}\right|+\left|b_{1 j}\right|\right)=4.9,2 M_{2}=\sum_{j=1}^{3}\left(\left|a_{2 j}\right|+\left|b_{2 j}\right|\right)$ $=5.3,2 M_{3}=\sum_{j=1}^{3}\left(\left|a_{3 j}\right|+\left|b_{3 j}\right|\right)=5.12$. Moreover, $k_{i}=h_{i}=1(i=1, \ldots, n)$. According to Theorem 4.1, the network (5.1) is globally exponentially stable in Lagrange sense. Furthermore, we can estimate the size of the global exponential attractors $\Omega_{1}, \Omega_{2}, \Omega_{3}$. If we select $\epsilon_{1}=0.5, \epsilon_{2}=1 / 10, \epsilon_{3}=1 / 20$ to satisfy $0<\epsilon_{i}<\underline{d_{i}}$, then

$$
\Omega_{1}=\left\{x \in \mathbb{R}^{3} \mid \frac{1}{2} \sum_{i=1}^{3} x_{i}^{2} \leq \frac{(2.45)^{2}+(2.65)^{2}+(2.56)^{2}}{0.5} \approx 39.1572\right\}
$$


is a GEA set of (5.1). Fig. 1 depicts the state trajectories for delay values $(0.01,0.02,0.01)$ and 12 initial values.
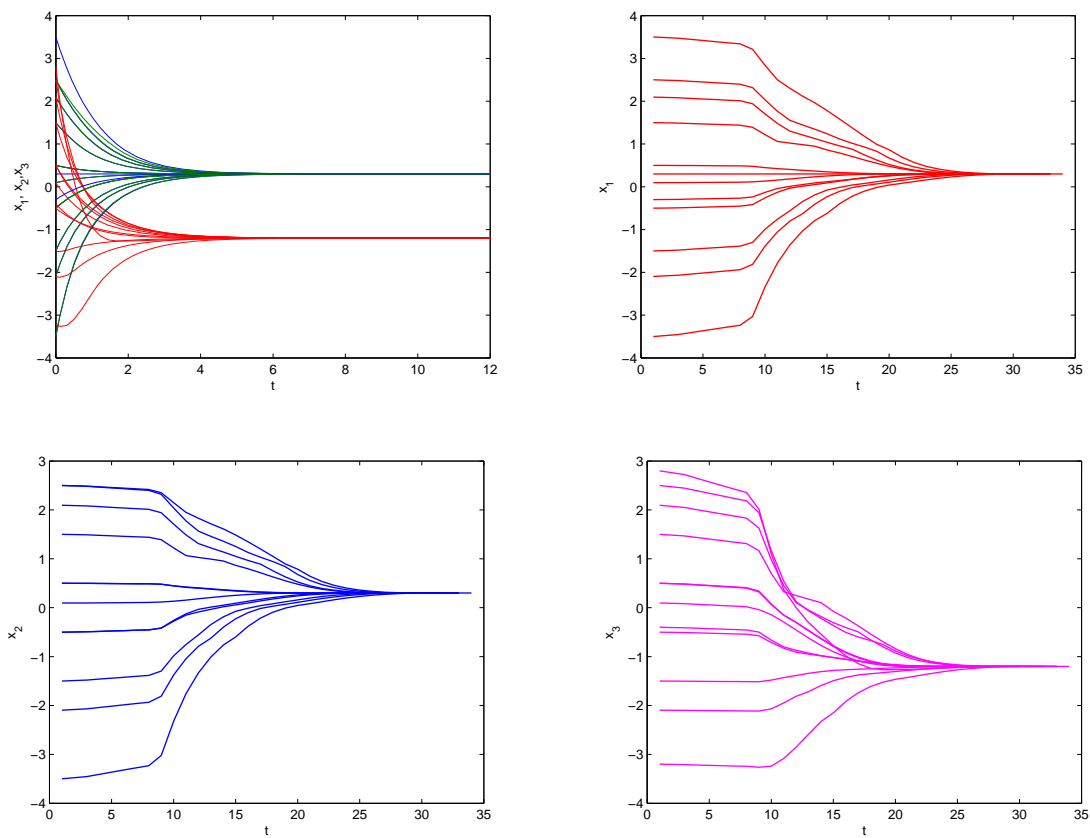

Fig. 1. State trajectories of solutions of 5.1 for $s=-0.8$

Similarly, $\Omega_{2}=\left\{\left(x_{1}, x_{2}, x_{3}\right)|| x_{1}|\leq 4.9,| x_{2}|\leq 5.3, \quad| x_{3} \mid \leq 5.12\right\}$ is also a globally exponentially attractive set of (5.1). Moreover, for $s=0$, $\Omega_{3}=\left\{\left(x_{1}, x_{2}, x_{3}\right)|| x_{1}|\leq 4.9,| x_{2}|\leq 6.9,| x_{3} \mid \leq 5.2\right\}$ is a globally exponentially attractive set of (5.1). In the same manner, we can construct other globally exponentially attractive sets.

EXAMPLE 5.2. Consider the above neural network model (5.1) with sigmoidal and bounded type activation functions. Take

$$
A=\left(\begin{array}{ccc}
-1 & -2 & 4 \\
2 & -2 & 3 \\
-4 & -3 & 8
\end{array}\right) \quad \text { and } \quad B=\left(\begin{array}{ccc}
-4 & -2 & 3 \\
2 & -1 & 8 \\
-3 & -8 & 7
\end{array}\right)
$$

with

$$
f(x)=(|x+1|-|x-1|) / 2, \quad g(x)=\frac{e^{x}-e^{-x}}{e^{x}+e^{-x}} ;
$$

then $a_{i j}, b_{i j}, c_{i j}$ are all constants. Take

$$
P=\left(\begin{array}{ccc}
0 & 0 & 0 \\
0 & 0 & 0 \\
0 & 0 & -8
\end{array}\right) \quad \text { and } \quad Q=\left(\begin{array}{ccc}
0 & 0 & 0 \\
0 & 0 & 0 \\
0 & 0 & -7
\end{array}\right) ;
$$


then

$$
\bar{A}=\left(\begin{array}{ccc}
-1 & -2 & 4 \\
2 & -2 & 3 \\
-4 & -3 & 0
\end{array}\right) \quad \text { and } \quad \bar{B}=\left(\begin{array}{ccc}
-4 & -2 & 3 \\
2 & -1 & 8 \\
-3 & -8 & 0
\end{array}\right)
$$

$\bar{A}+\bar{A}^{T} \leq 0$ and $\bar{B}+\bar{B}^{T} \leq 0$. By Theorem 3.6, the sets

$$
\begin{aligned}
& \Omega_{4}=\left\{x \in \mathbb{R}^{3} \mid \sum_{i=1}^{3} \int_{0}^{x_{i}(t)}\left(f_{i}(y)+g_{i}(y)\right) d y \leq 18^{2}+23^{2}\right\}, \\
& \Omega_{5}=\left\{x \in \mathbb{R}^{3} \mid \sum_{i=1}^{3} \int_{0}^{x_{i}(t)}\left(f_{i}(y)+g_{i}(y)\right) d y \leq 82\right\}
\end{aligned}
$$

are globally exponentially attractive for (5.1).

EXAMPLE 5.3. Consider the two-neuron network model described as:

$$
\begin{aligned}
& \dot{y}_{1}(t)=-0.45 y_{1}(t)-0.0489 f\left(y_{2}(t)\right)+0.0752 \sin (t) g\left(y_{2}(t-0.02)\right), \\
& \dot{y}_{2}(t)=-0.45 y_{2}(t)-0.0489 f\left(y_{1}(t)\right)+0.0752 \sin (t) g\left(y_{1}(t-0.01)\right) .
\end{aligned}
$$

We consider different bounded type activation functions in this case. Take $f(y)=\sin (y)$ and $g(y)=(|y+1|-|y-1|) / 2, \varepsilon_{1}=0.2, \varepsilon_{2}=0.3$. Then $h_{i}=g_{i}=1$.
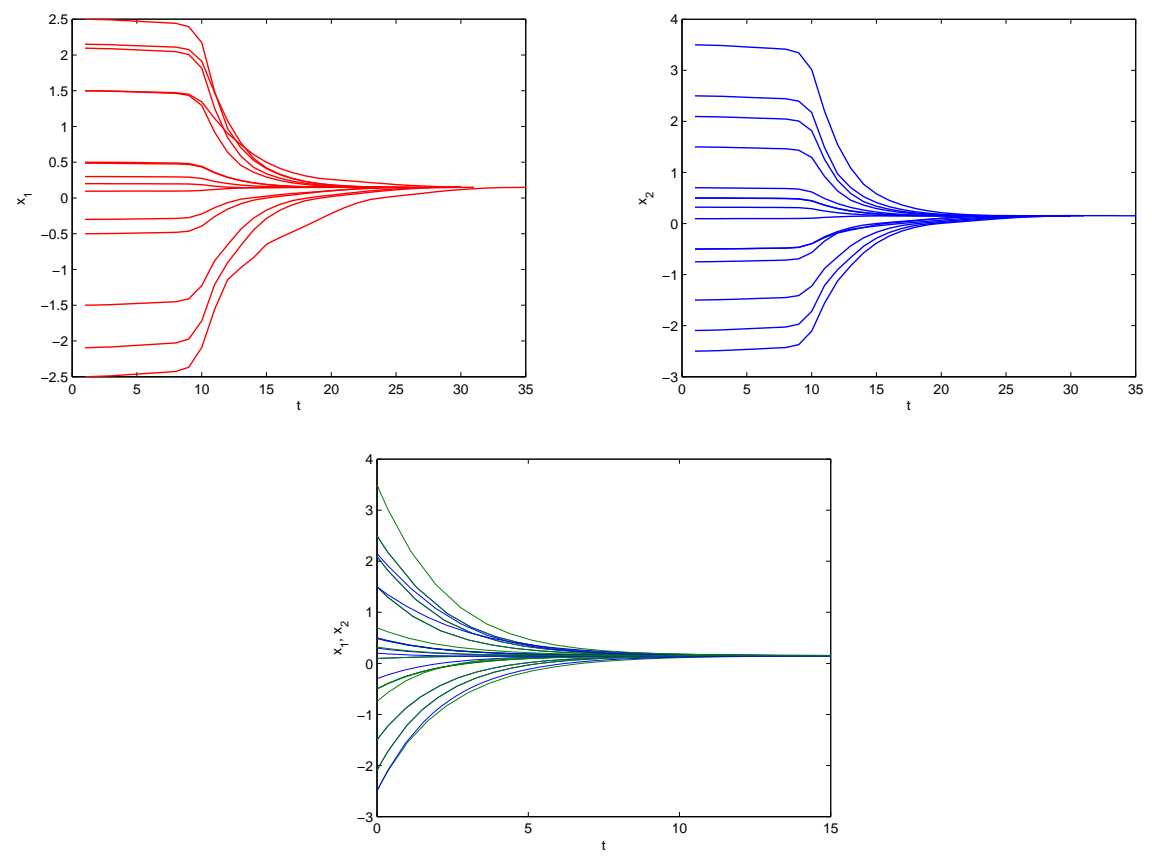

Fig. 2. State trajectories of solutions of system 5.2 
Clearly, the network (5.2) is globally exponentially stable in Lagrange sense, and from Theorem 3.1 we have the following GEA sets:

$$
\begin{aligned}
& \Omega_{1}=\left\{x \in \mathbb{R}^{2} \mid \frac{x_{1}^{2}(t)+x_{2}^{2}(t)}{2} \leq 0.78361\right\}, \\
& \Omega_{2}=\left\{x \in \mathbb{R}^{2}|| x_{1}(t)|\leq 1.2538,| x_{2}(t) \mid \leq 1.2538\right\}, \\
& \Omega_{3}=\left\{x \in \mathbb{R}^{2}|| x_{1}(t)|+| x_{2}(t) \mid \leq 2.5076\right\} .
\end{aligned}
$$

A simulation result for the network $(5.2)$ with 15 initial values is depicted in Fig. 2.

6. Conclusion. In this work, we have studied the global exponential stability in Lagrange sense for periodic neural networks with general bounded and sigmoidal activation functions and multiple time-varying delays, relying on Lyapunov stability theory. Via analyzing different activation functions and constructing different Lyapunov functionals, we have obtained several globally exponentially attractive sets. Moreover, we have verified that there is no periodic state, almost periodic state or chaos attractor outside the globally exponentially attractive set. Since we have not made any assumption on the number of equilibria, the results obtained can be applied to analyzing monostable, multistable as well as more extensive neural networks. From the comparison between different attractive sets $\Omega_{i}$, we find a better method to reduce the constraints on the criteria, which narrows the search domains of optimization and associative memories and also provides theoretical guidelines for applications. The results are applicable to monostable and multistable neural networks as well as chaos control and chaos synchronization and can be extended to more complex systems.

Acknowledgements. We are grateful to the editor, associate editor and anonymous reviewers for their insightful comments and suggestions, which helped us improve the manuscript considerably.

\section{References}

[1] S. Abbas, Existence and attractivity of k-pseudo almost automorphic sequence solution of a model of bidirectional neural networks, Acta Appl. Math. 23 (2012), 57-74.

[2] S. Abbas, L. Mahto, M. Hafayed and A. M. Alimi, Asymptotic almost automorphic solutions of impulsive neural network with almost automorphic coefficients, Neurocomputing 142 (2014), 326-334.

[3] M. Akhmet and E. Ylmaz, Neural networks with discontinuous/impact activations, Springer, New York, 2014.

[4] T. A. Burton, Stability and Periodic Solutions of Ordinary and Functional Differential Equations, Courier Corporation, 2005. 
[5] K. S. Chiu, M. Pinto and J. C. Jeng, Existence and global convergence of periodic solutions in recurrent neural network models with a general piecewise alternately advanced and retarded argument, Acta Appl. Math. 133 (2014), 133-152.

[6] K. Gopalsamy, Stability and Oscillations in Delay Differential Equations of Population Dynamics, Springer, 1992.

[7] E. Gyftopoulos, Lagrange stability by Liapunov's direct method, in: Reactor Kinetics and Control, TIE Washington, 1964, 227-237.

[8] J. J. Hopfield, Neurons with graded response have collective computational properties like those of two-state neurons, Proc. Nat. Acad. Sci. USA 81 (1984), 3088-3092.

[9] H. F. Huo, W. T. Li and S. Tang, Dynamics of high-order BAM neural networks with and without impulses, Appl. Math. Comput. 215 (2009), 2120-2133.

[10] H. Jiang and Z. Teng, Boundedness, periodic solutions and global stability for cellular neural networks with variable coefficients and infinite delays, Neurocomputing 72 (2009), 2455-2463.

[11] X. Li, Existence and global exponential stability of periodic solution for impulsive Cohen-Grossberg-type BAM neural networks with continuously distributed delays, Appl. Math. Comput. 215 (2009), 292-307.

[12] Y. Li, X. Chen and L. Zhao, Stability and existence of periodic solutions to delayed Cohen-Grossberg BAM neural networks with impulses on time scales, Neurocomputing 72 (2009), 1621-1630.

[13] X. Liao, Q. Luo and Z. Zeng, Positive invariant and global exponential attractive sets of neural networks with time-varying delays, Neurocomputing 71 (2008), 513-518.

[14] X. Liao, Q. Luo, Z. Zeng and Y. Guo, Global exponential stability in Lagrange sense for recurrent neural networks with time delays, Nonlinear Anal. Real World Appl. 9 (2008), 1535-1557.

[15] X. Liao and Z. Zeng, Global exponential stability in Lagrange sense of continuoustime recurrent neural networks, Adv. Neural Networks-ISNN, Springer, Berlin, 2006, $115-121$.

[16] Q. Luo, Z. Zeng and X. Liao, Global exponential stability in Lagrange sense for neutral type recurrent neural networks, Neurocomputing 74 (2011), 638-645.

[17] M. Pinto and G. Robledo, Existence and stability of almost periodic solutions in impulsive neural network models, Appl. Math. Comput. 217 (2010), 4167-4177.

[18] S. Tyagi, S. Abbas and M. Kirane, Global asymptotic and exponential synchronization of ring neural network with reaction-diffusion term and unbounded delay, Neural Computing Appl. 30 (2018), 487-501.

[19] S. Tyagi, S. Abbas and R. K. Ray, Stability analysis of an integro differential equation model of ring neural network with delay, in: Mathematical Analysis and its Applications, Springer, New Delhi, 2015, 37-49.

[20] B. Wang, J. Jian and M. Jiang, Stability in Lagrange sense for Cohen-Grossberg neural networks with time-varying delays and finite distributed delays, Nonlinear Anal. Hybrid Systems 4 (2010), 65-78.

[21] X. Wang, M. Jiang and S. Fang, Stability analysis in Lagrange sense for a nonautonomous Cohen-Grossberg neural network with mixed delays, Nonlinear Anal. 70 (2009), 4294-4306.

[22] A. Wu, Z. Zeng, C. Fu and W. Shen, Global exponential stability in Lagrange sense for periodic neural networks with various activation functions, Neurocomputing 74 (2011), 831-837.

[23] A. Wu and Z. Zeng, Lagrange stability of neural networks with memristive synapses and multiple delays, Information Sci. 280 (2014), 135-151. 
[24] H. Wu and C. Shan, Stability analysis for periodic solution of BAM neural networks with discontinuous neuron activations and impulses, Appl. Math. Modelling 33 (2009), $2564-2574$.

[25] X. Yang, Existence and global exponential stability of periodic solution for CohenGrossberg shunting inhibitory cellular neural networks with delays and impulses, Neurocomputing 72 (2009), 2219-2226.

[26] Z. Yi and K. K. Tan, Convergence Analysis of Recurrent Neural Networks, Network Theory Appl. 13, Kluwer, 2004.

[27] J. Zhang and Z. Gui, Periodic solutions of nonautonomous cellular neural networks with impulses and delays, Nonlinear Anal. Real World Appl. 10 (2009), 1891-1903.

Swati Tyagi

Indian Institute of Technology Ropar

Rupnagar 140001, India

E-mail: styagi99@gmail.com

Manuel Pinto

Departamento de Matemáticas

Facultad de Ciencias

Universidad de Chile

Santiago, Chile

E-mail: pintoj.uchile@gmail.cl
Syed Abbas (corresponding author) Indian Institute of Technology Mandi Mandi 175005, India E-mail:sabbas.iitk@gmail.com 